\title{
On the Way to Solve Lighting Problems in Underwater Imaging
}

\author{
Rafael Garcia, Tudor Nicosevici and Xevi Cufí \\ Computer Vision and Robotics Group \\ Institute of Informatics and Applications \\ University of Girona, E.P.S. \\ 17071 Girona, Spain \\ e-mail: \{rafa,tudor,xcuf\}@eia.udg.es
}

\begin{abstract}
A major obstacle to processing images of the ocean floor comes from the absorption and scattering effects of the light in the aquatic environment. Due to the absorption of natural light, underwater vehicles often require artificial light sources attached to them to provide the adequate illumination. Unfortunately, these flashlights tend to illuminate the scene in a nonuniform fashion, and, as the vehicle moves, induce shadows in the scene. For this reason, the first step towards application of standard computer vision techniques to underwater imaging requires dealing first with these lighting problems. This paper analyses and compares existing methodologies to deal with lowcontrast, nonuniform illumination in underwater image sequences. The reviewed techniques include: $(i)$ study of the illumination-reflectance model, (ii) local histogram equalization, (iii) homomorphic filtering and (iv) subtraction of the illumination field. Several experiments on real data have been conducted to compare the different approaches.
\end{abstract}

\section{INTRODUCTION}

In the last few years Unmanned Underwater Vehicles (UUVs) have been used to survey the ocean floor. Optical sensors are being introduced in these vehicles either to improve local navigation $[1,2]$ or to provide a global view of the site of interest [3]. In both cases, automatic image processing systems become an important tool for ocean exploration [4].

A major obstacle to processing images of the ocean floor is related to the special transmission properties of the light in the underwater medium [5]. Light suffers two different processes in the aquatic environment [6]: ( $i$ ) absorption, where light disappears from the image-forming process, and (ii) scattering, a change of direction of the individual photons, mainly due to the different sizes of the particles forming the water. These transmission properties of the medium generate some problems in underwater images, such as blurring of image features, limited range, clutter and lack of structure in the regions of interest. In some cases, small observable particles floating in the water are imaged as "marine snow", precluding subsequent processing. And often, natural light is not sufficient for imaging the sea floor. For this reason, a light source is normally attached to the submersible providing the necessary lighting. Artificial light sources not only suffer from the difficulties described before (i.e. scattering, absorption, etc.), but in addition tend to illuminate the scene in a nonuniform fashion, producing a bright spot in the center of the image with a poorly illuminated area surrounding it. Moreover, the motion of the light source creates a shift of the shadows induced in the scene, generating a change in the brightness of the imagery as the vehicle moves. For all these reasons, application of standard computer vision techniques to underwater imaging requires dealing first with these added problems.

In this paper we carry out a first step towards comparing existing methodologies to deal with low-contrast, nonuniform illumination in underwater imaging. First, section II provides a review of image enhancement techniques which aim to compensate these lighting effects. Some of the approaches have been slightly modified to adapt them to the peculiarities of the underwater environment. Then, a set of experiments performed on real data is shown in section III, and, finally, the conclusions close the paper.

\section{COMPENSATION OF THE LIGHTING EFFECTS}

In this section, various strategies for correcting nonuniform lighting are reviewed: (i) exploitation of the illumination-reflectance model, (ii) local histogram equalization, (iii) homomorphic filtering and (iv) subtraction of the illumination field by polynomial adjustment. The statement of the problem would be as follows. Given a grayscale image which presents lighting inhomogeneities, taken by an underwater vehicle, how can we enhance it so that the result is more suitable than the original image for further processing.

\section{A. Illumination-Reflectance Model}

This approach considers the image as a function of the product of the illumination and reflectance properties of a given scene [7], as described by equation (1).

$$
f(x, y)=i(x, y) \cdot r(x, y),
$$

where $f(x, y)$ is the image sensed by the camera, $r(x, y)$ is the reflectance function (or ideal image under absence of shading) and $i(x, y)$ represents the illumination multiplicative 
factor. Depending upon the camera characteristics, it may also contribute gain $g(x, y)$ and offset $o(x, y)$ terms:

$$
f(x, y)=g(x, y) \cdot i(x, y) \cdot r(x, y)+o(x, y),
$$

Therefore, equation (2) can be expressed as a reflectance function adjusted by a multiplicative $c_{m}(x, y)$ and an additive $c_{a}(x, y)$ shading component:

$$
f(x, y)=c_{m}(x, y) \cdot r(x, y)+c_{a}(x, y),
$$

Normally, the multiplicative factor $c_{m}(x, y)$ due to light sources carried by the vehicle and camera sensitivity can be modeled as a smooth function. In order to model this nonuniform illumination, a Gaussian-smoothed version of the image acquired by the camera $f(x, y)$ is proposed. The smoothed image $f_{s}(x, y)$ is intended to be an estimate of how much the illumination field (and camera sensitivity) affects every pixel of the image. To obtain this effect, the smoothing has to be large compared to the size of the features in the image. Therefore, the acquired image can be corrected by a point-by-point division by the smoothed image, giving rise to an estimate of ideal image $\tilde{r}(x, y)$ can be obtained through:

$$
\tilde{r}(x, y)=\frac{f(x, y)}{f_{s}(x, y)} \cdot \delta,
$$

where $\delta$ is a normalization constant which restores the overall image luminance. Here we have deliberately ignored offset term $c_{a}(x, y)$ which could come from non-uniform camera sensitivity since in an underwater environment its influence is very small with respect to $i(x, y)$, at least for a standard camera.

Next, the contrast of the resulting image is emphasized through equation (5), giving rise to $\tilde{r}^{\prime}(x, y)$, an "equalized" version of $\tilde{r}(x, y)$.

$$
\tilde{r}^{\prime}(x, y)=[\tilde{r}(x, y)-\min (\tilde{r}(x, y))] \frac{\tau_{H}-\tau_{L}}{\max (\tilde{r}(x, y))-\min (\tilde{r}(x, y))}+\tau_{L},
$$

where $\tau_{H}$ and $\tau_{L}$, are, respectively, the maximum and minimum desired values of $\tilde{r}^{\prime}(x, y) ; \max (\tilde{r}(x, y))$ represents the brightest gray level of image $\tilde{r}(x, y)$ which is smaller than 1.5 times the third quartile of the histogram of $\tilde{r}(x, y)$; and a similar approach is applied to $\min (\tilde{r}(x, y))$ with the first quartile. In this way the strategy increases its robustness with respect to noise.

Moreover, in our implementation the smoothed image is not computed for every image of the sequence. It is only computed from a set of consecutive frames. The result is averaged and then a 2D-Gaussian function is adjusted to the average image. In this way, it is used in equation (4) for every new image, thus saving computational effort.

\section{B. Local Histogram Equalization and Its Variations}

The histogram of an image encodes information about the nature of this image. Histogram-modeling techniques modify the image so that its histogram has some desired shape. In histogram equalization, the goal is to enhance the image so that an "optimal" overall contrast is obtained. However, in underwater imaging, the nonuniform nature of the lighting demands a different treatment of the various different areas of the image, depending on the amount of light they receive. For this reason, some authors compensate for the effects of non-uniform lighting by applying local equalization to the images $[3,8]$. This strategy consists in defining an $n \times n$ neighborhood, computing the histogram of this area, and applying an equalization function, but modifying uniquely the central point of the neighborhood [9]. This operation is then repeated for all the pixels of the image. When one applies this technique to images suffering from non-uniform lighting, a more balanced image is obtained. Unfortunately, local equalization is very time consuming and, although various algorithms have been devised to make it more efficient, it is still inadequate for real-time applications. Moreover, it has a tendency to amplify noise in poorly contrasted areas.

Other works propose the use of a similar strategy to enhance the contrast in underwater images [10]: Contrast Limited Adaptive Histogram Equalization (CLAHE). This technique, described in [11], subdivides the image into $n \times m$ blocks, calculating the histogram of each such block. Every region is then equalized by choosing a monotonically nondecreasing gray level transformation, mapping the histogram of a desired distribution. However, the enhancement of a block is limited by the selection of a clipping level, defined as a multiple of the histogram average. Those pixels exceeding the clip limit are equally redistributed across the histogram, their value being finally adjusted according to interpolation between the histograms of neighboring regions. The clipping factor may help, if properly adjusted, to reduce noise amplification in poorly contrasted areas.

\section{Homomorphic Filtering}

Another common technique which takes into account the illumination-reflectance model of equation (3) consists of applying homomorphic filtering [12]. This approach takes into account uniquely the multiplicative term $c_{m}(x, y)$, considering $c_{a}(x, y)=0$. It assumes the illumination factor varies smoothly through the field of view, therefore generating low frequencies in the Fourier transform of the image. On the other hand, reflectance is associated with the high frequency components of the image. Homomorphic filtering separates both factors by taking the logarithm of equation (3), as shown in equation (6). This equation is the 
basis of the filter, since it converts the multiplicative effect into an additive one, allowing the separation of both components.

$$
\ln f(x, y)=\ln c_{m}(x, y)+\ln r(x, y),
$$

Taking the Fourier transform of equation (6), we obtain:

$$
F\left(\omega_{x}, \omega_{y}\right)=C_{m}\left(\omega_{x}, \omega_{y}\right)+R\left(\omega_{x}, \omega_{y}\right),
$$

where $F\left(\omega_{x}, \omega_{y}\right), C_{m}\left(\omega_{x}, \omega_{y}\right)$ and $R\left(\omega_{x}, \omega_{y}\right)$ are the Fourier transforms of $\ln f(x, y), \ln c_{m}(x, y)$ and $\ln r(x, y)$, respectively. Then, low frequencies are suppressed by multiplying these components by a high-pass homomorphic filter $H(\cdot)$ :

$$
\begin{aligned}
& H\left(\omega_{x}, \omega_{y}\right) F\left(\omega_{x}, \omega_{y}\right) \\
& =H\left(\omega_{x}, \omega_{y}\right) C_{m}\left(\omega_{x}, \omega_{y}\right)+H\left(\omega_{x}, \omega_{y}\right) R\left(\omega_{x}, \omega_{y}\right) .
\end{aligned}
$$

To go back to the spatial domain, we compute the Inverse Fourier transform of equation (8), and then taking the exponent, the enhanced image can be obtained.

The filter not only attenuates nonuniform illumination, but also enhances the high frequencies, sharpening the edges of the objects in the image. Equation (9) shows a typical transfer function for the homomorphic filter [13]. The shape of the filter is shown in Fig. 1.

$$
H\left(\omega_{x}, \omega_{y}\right)=\frac{1}{1+e^{-s\left(\sqrt{\omega_{x}^{2}+\omega_{y}^{2}}-\omega_{0}\right)}}+\rho,
$$

where $\omega_{0}$ is the cutoff frequency, $s$ is a multiplicative factor and $\rho$ is an offset term.

\section{Subtraction of the Illumination Field}

The approach presented in [14] for removal of lighting inhomogeneities could be considered to derive from the illumination-reflectance model presented above in equation (3). When we described our implementation of this model in section II. $A$, we assumed the offset term $c_{a}(x, y)$ could be neglected with respect to the multiplicative shading component $c_{m}(x, y)$. On the contrary, the strategy proposed in [14] disregards the multiplicative component, considering the lighting of the scene as an additive factor which should

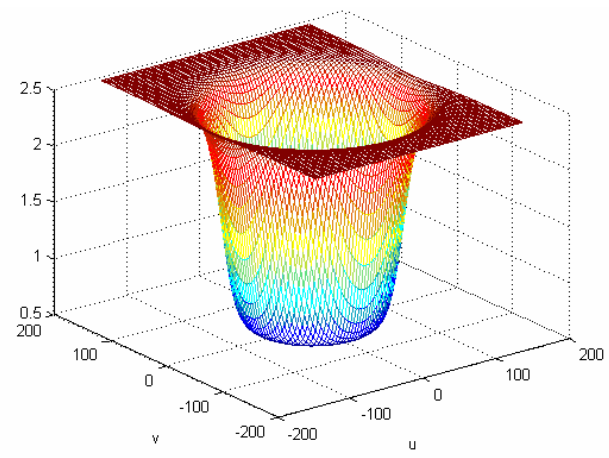

Fig. 1. Homomorphic filter $H\left(\omega_{x}, \omega_{y}\right)$. be subtracted from the original image. The proposed technique consisted in fitting a low-order two-dimensional polynomial spline to every frame $\Phi(x, y)$ and then subtract it from the acquired image $f(x, y)$ :

$$
\tilde{r}(x, y)=f(x, y)-\Phi(x, y)+\delta
$$

where $\delta$ is a normalization constant needed to adjust the desired luminance. We should bear in mind that, although illumination acts as a multiplicative factor in the image formation process, shading effects are also affected by the camera characteristics. Determining which shading component should be considered involves a study of the image formation model for a specific sensor, e.g. background subtraction has been successfully applied to microscopy images for the last few years. However, for our experience in underwater images, the multiplicative component $c_{m}(x, y)$ has quite a relevant effect. In any case, subtraction of the parametric surface $\Phi(x, y)$ from an image presenting nonuniform illumination results in darkening the center of the image and lighting the poorly illuminated zones of the sides. The coefficients of the spline can be obtained through averaging over several frames.

\section{EXPERIMENTAL RESULTS}

Some experiments have been performed to compare the different lighting-correction strategies. Two typical underwater situations have been used to test the approaches reviewed above. The first one considers images with serious non-uniform effects. In these images, the vehicle carries its own light producing a bright spot close to the center of the image. The underwater terrain presents a flat area with sand and rocks. The images have been acquired in shallow waters at sundown, to avoid the influence of the sun rays, thus simulating Deep Ocean. The second sequence of images presents a different difficulty. They have been acquired in shallow waters on a sunny day. The underwater terrain presents some small 3D relief. In this case the aim is to see if the various approaches can cope with the lighting artifacts produced by sun rays deflected by the waves. Our interest in such images comes from the fact that some of our experiments have to be performed in shallow waters. Therefore, we are also interested in the behavior of image enhancement methodologies under these circumstances.

Fig. 2 (left) shows three images of an underwater sequence. The original size of the images is $384 \times 288$ pixels. Smoothing these images with a large Gaussian kernel (of size $63 \times 63$ ) gives rise to the images displayed in Fig. 2 (right). The size of the smoothed images has been kept constant. This explains the gray border around the filtered images. 

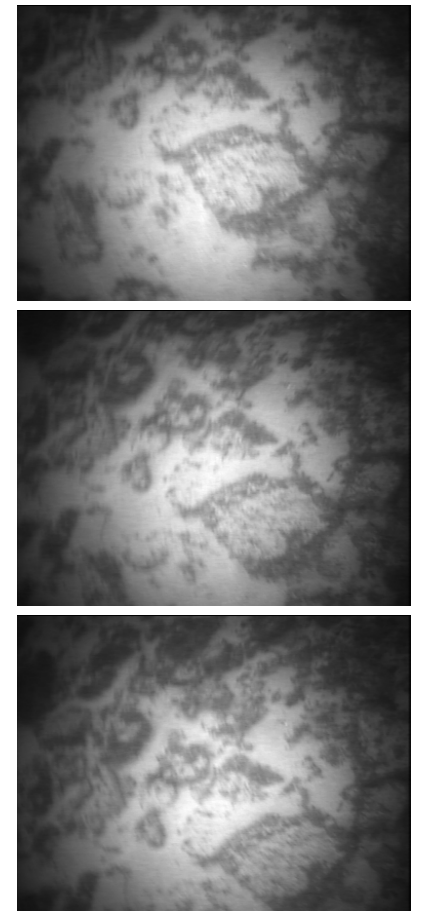

(a)
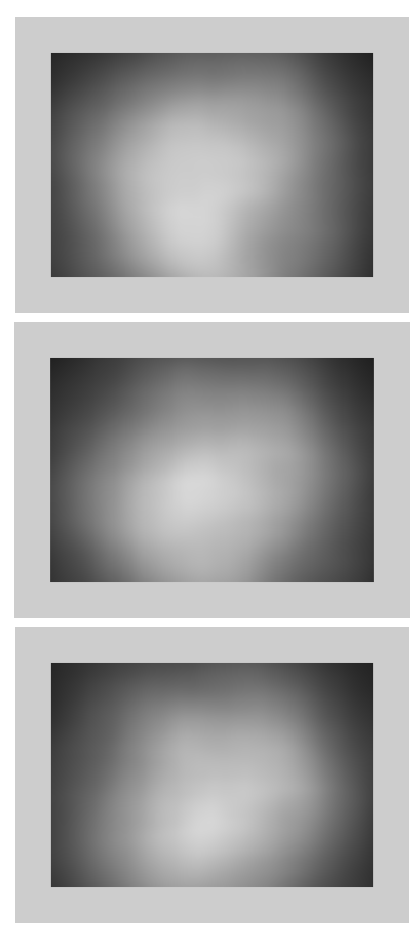

(b)

Fig. 2. Estimation of the illumination field in a sequence of images presenting nonuniform illumination. (a) Original images, (b) Result of low-pass filtering with a Gaussian kernel of size $63 \times 63$ pixels and $\sigma=41$.

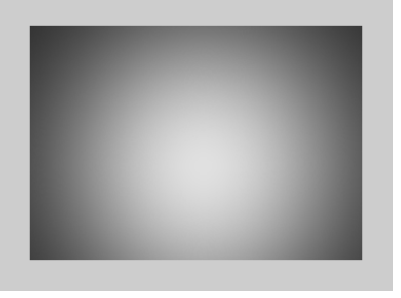

Fig. 3. Resulting image after adjusting a Gaussian function to a set of smoothed images.

In most of the strategies several parameters have to be manually selected to obtain the enhanced images. In all cases we have tuned the parameters taking into account two main factors: (1) correction of the lighting inhomogeneities, and (2) similarity of consecutive images of the sequence. This means that we have searched the trade-off between both aspects. The second one is very important if further processing aims to measure some parameter between consecutive images, (e.g. motion detection). For this reason, although some individual images had a more "natural" aspect with a different parameter adjustment, the tuning which made the sequence look uniform was selected. Our evaluation methodology is based uniquely in qualitative measurements performed by the authors. This means that there may exist a certain degree of subjectivity in the evalua-

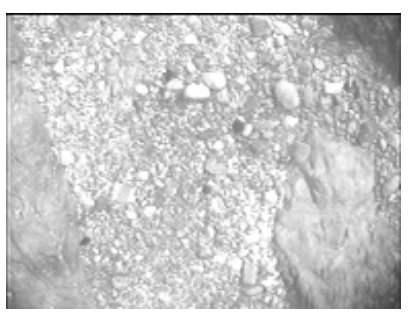

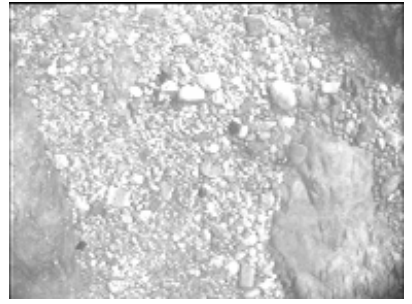

(a)
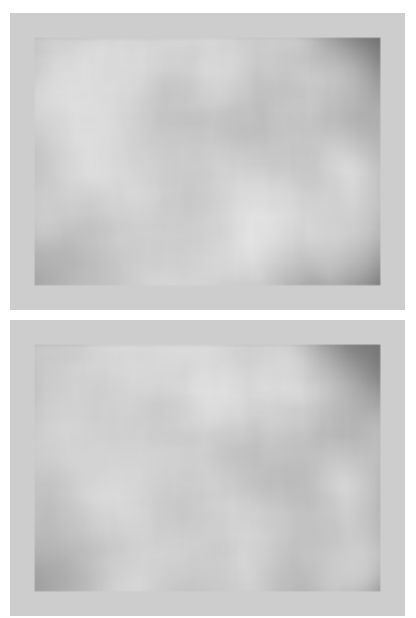

(b)
Fig. 4. Correction of non-uniform lighting. (a) Original images, (b) Result of low-pass filtering with a Gaussian kernel of size $25 \times 25$ pixels and $\sigma=15$.

tion. For this reason, in this comparative study we will solely stress the main aspects of every technique.

The result of averaging several smoothed images, and then adjustment of a Gaussian function is illustrated in Fig. 3. Fig. 4 (left) shows the second set of test frames: a pair of images suffering from lighting inhomogeneities generated by the deflection of sun rays. These images are $192 \times 144$ pixels. On the right, Fig. 4 shows the result of convolving the images with a $25 \times 25$ Gaussian filter.

Figs. 5(a) and 6(a) show the result of dividing the original image by the smoothed one, following the illuminationreflectance model. For all the frames of the first sequence, the image of Fig. 3 has been taken as smoothed image $f_{s}(x, y)$. In fact, the resulting enhanced images for the first sequence compensate the nonuniform lighting in a quite efficient way. In the case of the second sequence (see Fig. 6(a)), the shading due to the waves has been slightly attenuated, though it is still perceptible. In this case, the smoothed images have not been averaged and no function has been adjusted to these images, since shading changes rapidly from one image to the next. Therefore, the result of the illumination-reflectance model which considers the multiplicative factor of the shading is not as good for the second sequence as it is for the first one, but it is still acceptable. However, the images of Fig. 6(a) are more adequate for subsequent processing than the original ones.

Figs. 5(b) and 6(b) show the result of applying local equalization to both image sequences. The resulting images are not very adequate to be further processed. The first sequence looks a bit overexposed. There is significant difference from one image to the next within the same sequence. Some of the uniform sandy areas, which were 

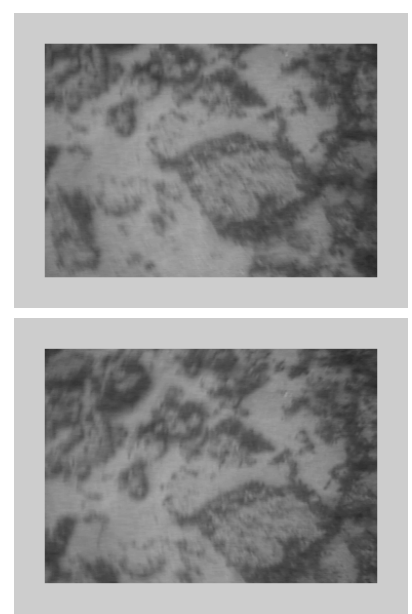

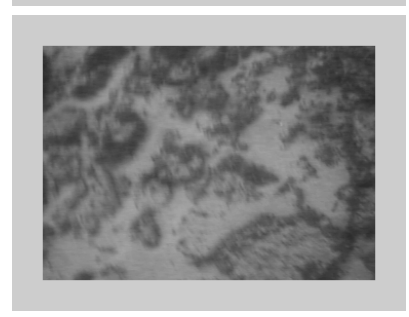

(a)
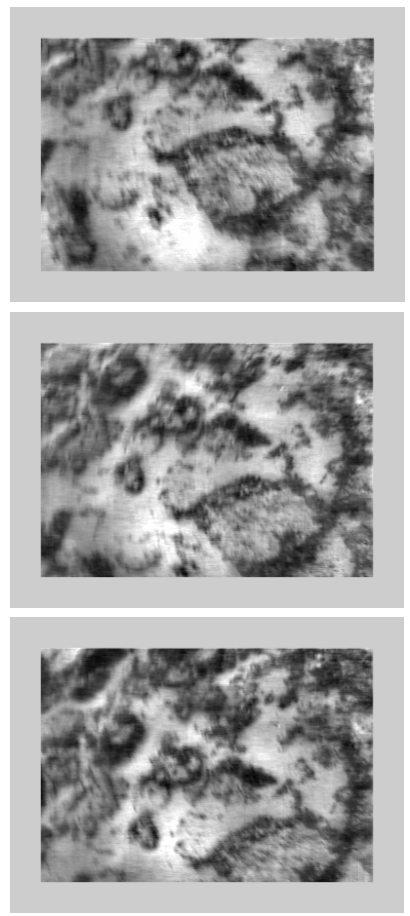

(b)
Fig. 5. Correction of non-uniform lighting. (a) Images enhanced through the Illumination-Reflectance model (multiplicative approach), using the Gaussian function illustrated in Fig. 3; (b) Local histogram equalization considering a $61 \times 61$ neighborhood.

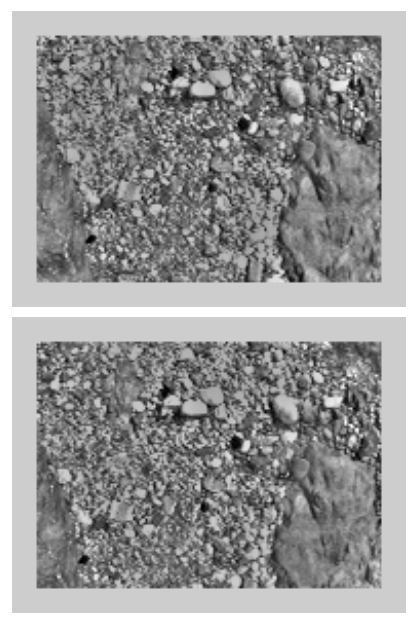

(a)
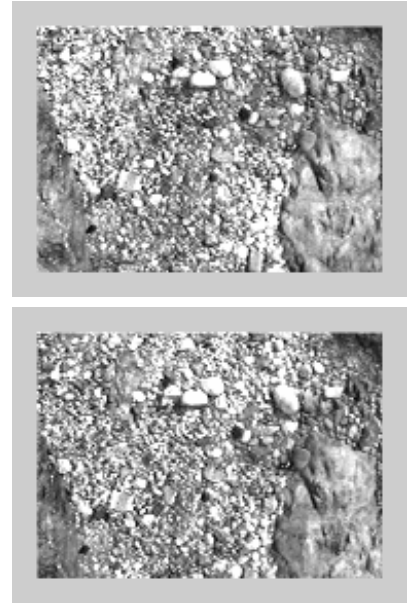

(b)
Fig. 6. Correction of lighting artifacts generated by the waves. (a) Images corrected through the Illumination-Reflectance model (multiplicative approach), considering the Gaussian functions of Fig. 6 (left); (b) Local histogram equalization considering a $25 \times 25$ neighborhood.

quite homogeneous in the original image, present considerable variations in their apparent texture. Local equalization tends to amplify noise in these poor-contrasted areas. In the second sequence, the shading induced by the waves is more noticeable than in the previous method (illumination-reflectance model). Moreover, some areas of the top image (Fig. 6(b)) have become darker than those same areas in the bottom image (look at the central stripe and the top right hand side of the image).

On the other hand, Contrast Limited Adaptive Histogram Equalization (CLAHE) can be parameterized so that it presents fewer differences among images of the same sequence. This can be clearly observed in Fig. 7(a). However, we can also see how the equalization performed by CLAHE de-emphasizes small regions of sharp edges [9], while simultaneously increasing the size of these regions. In the second sequence, the results are a lot better than those obtained through local equalization. However, some reflections are still perceptible, mainly in the rock on the bottom right of the images. The central part of the frames of Fig. 8(a) present different gray levels between top and bottom images.

The frames of Figs. 7(b) and 8(b) show the result of applying homomorphic filtering to the original image sequences. Again, the parameters of the filter have been adjusted to obtain similar-looking images within the sequence. Although none of the images has a natural appearance, both sequences succeed in both correcting lighting inhomogeneities and obtaining a quite similar aspect in the respective sequences. In this case the results show an increase in image contrast which has not been affected by the illumination artifacts of the original sequences.

Finally, the results obtained by subtracting the illumination field are presented in Fig. 9. Looking at the first sequence (left), we can see that the images are acceptably compensated for the excess of light in their central area. However, the border of these images does not obtain the necessary offset values to correct for the lack of light. In [14] this problem is solved by taking cut-off portions of the central part of images for further processing. In the second sequence, the shading effects are neither corrected. A possible explanation for this poor behavior may be in the fact that the multiplicative shading factor $c_{m}(x, y)$ has a much bigger influence in the image formation process than the additive one $c_{a}(x, y)$, in what concerns our imaging system.

\section{CONCLUSIONS AND FUTURE WORK}

It has been shown in this paper that a first step towards correction of lighting inhomogeneities can be achieved in the presence of nonuniform illumination when the ocean floor has a planar relief. The best results have been obtained by the two methods which consider the illumination field is multiplicative: homomorphic filtering and exploitation of the 

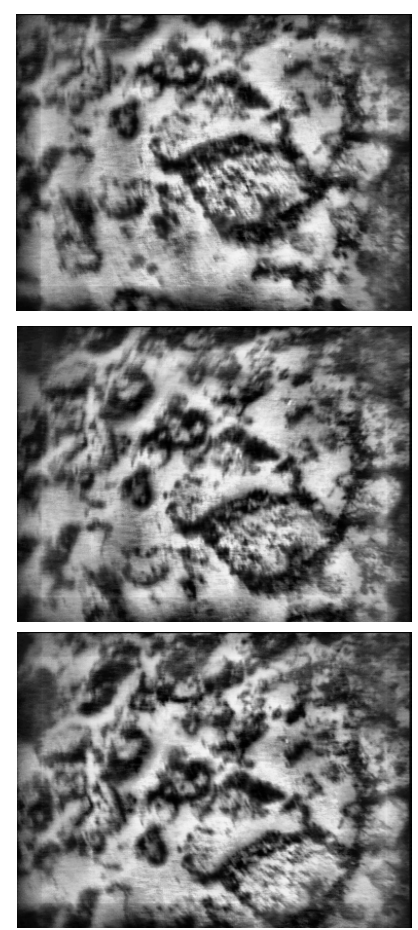

(a)

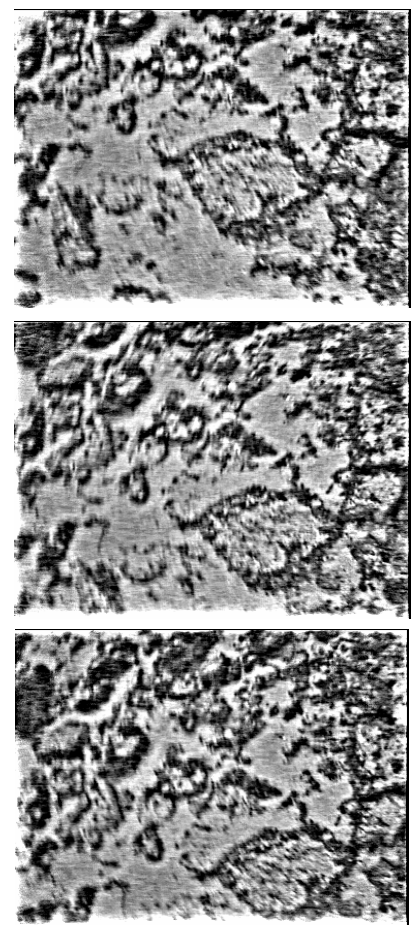

(b)

Fig. 7. Correction of non-uniform lighting. (a) Resulting sequence after enhancement applying Contrast Limited Adaptive Histogram Equalization (CLAHE); (b) Result obtained through homomorphic filtering.

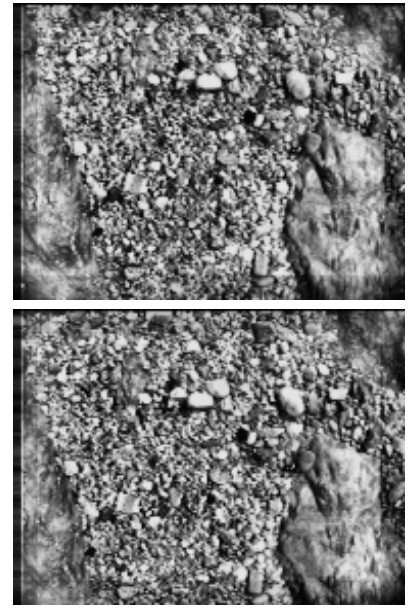

(a)
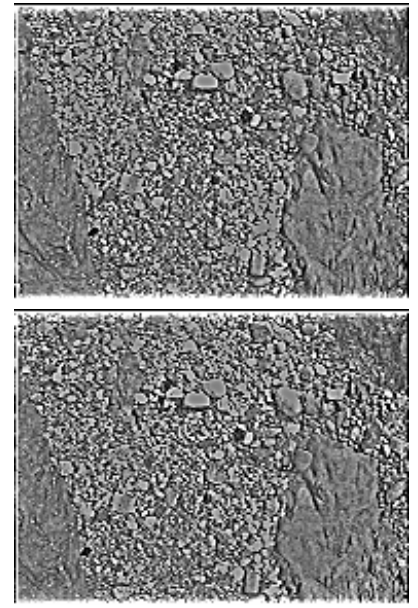

(b)
Fig. 8. Correction of lighting artifacts generated by the waves. (a) Contrast Limited Adaptive Histogram Equalization (CLAHE); (b) Images corrected through homomorphic filtering.

illumination-reflectance model, neglecting the additive component of the shading field. Processing of shallow water imaging has also been tested. This type of imagery presents a higher degree of difficulty. In this case, sun rays are refracted by the waves and image irradiance is irregularly concentrated in small areas of the image, and changes are evident from one image to the next. In this case results are not so satisfactory, but still promising for the homomorphic filter, and acceptable for the illumination-reflectance model.

Presence of small 3D relief has timidly been tested. It appears in this second sequence, although shadows originated by $3 \mathrm{D}$ objects of the underwater terrain (e.g. the rock on the right in Fig. 4) do not have a significant movement between images. In the future, this test will be performed with the underwater vehicle carrying out its own light source, thus producing shadows moving in opposite direction of the light source.

Homomorphic filtering assumes a separation of the high frequencies containing the true image data from the lower frequencies of the shading component. However, although this assumption has been valid in our test sequences, we may find some underwater images with an overlapping of the frequency spectra of the shading component and the image data, mainly due to the considerable amount of low frequency information some underwater images. Further tests should be performed in the future to verify the good behavior of the filter in all situations.

On the other hand, local equalization techniques, though acceptable in some cases, tend to amplify noise in poorcontrasted areas. Contrast Limited Adaptive Histogram Equalization (CLAHE) introduces more flexibility in the selection of the local histogram mapping function, and the clipping level of the histogram may help in the reduction of undesired noise amplification.

In the tested sequences, subtraction of the illumination field did not achieve very satisfying results. This is probably due to a domination of the multiplicative shading component our imaging system. However, future directions suggest searching for a model which takes into account both the multiplicative and the additive factors of the linear model of image formation, to check if an improvement can be obtained.

After this first step, more extensive experimentation should be performed to test the performance of the various approaches in the presence of 3D structure when the vehicle carries its own light, studying situations with strong shadows induced in the scene.

Finally, a methodology to perform quantitatively measures of the performance of every approach should be investigated.

\section{REFERENCES}

[1] S. Negahdaripour and J. Fox, "Underwater optical stationkeeping: improved methods", Journal of Robotic Systems, vol. 8, no. 3, pp. 319-338, 1991. 

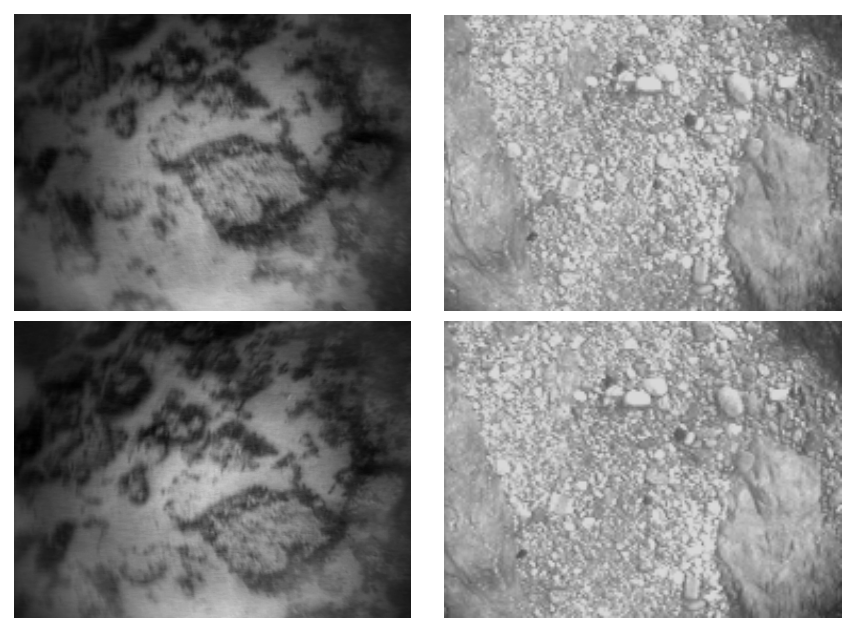

(a)

(b)

Fig. 9. Result of nonuniform illumination removal by subtraction of the additive shading effect. (a) First image sequence; (b) second image sequence

[2] N. Gracias and J. Santos-Victor, "Underwater Video Mosaics as Visual Navigation Maps," Computer Vision and Image Understanding, vol. 79, no. 1, pp. 66-91, 2000.

[3] H. Singh, J. Howland, D. Yoerger and L. Whitcomb, "Quantitative photomosaicing of underwater imaging," in Proc. MTS/IEEE OCEANS, vol. 1, pp. 263-266, 1998.

[4] R. Garcia, J. Batlle, X. Cufi, and J. Amat, "Positioning an Underwater Vehicle through Image Mosaicking," in Proc. IEEE Int. Conf. on Robotics and Automation, Seoul, Korea, vol. 3, pp. 2779-2784, 2001.
[5] C. J. Funk, S.B. Bryant and P.J. Beckman Jr., "Handbook of underwater imaging system design," Ocean Technology Department, Naval Undersea Center, 1972.

[6] S. Negahdaripour and C.H. Yu, "On shape and range recovery from image shading for underwater applications," in J. Yuh (ed.), Underwater Robotic Vehicles: design and control, ch. 8, pp. 221-250, TSI Press, 1995.

[7] R.C. Gonzalez and R.E. Woods. Digital Image Processing. Addison-Wesley, Reading, MA, 1992.

[8] R. Eustice, H. Singh and J. Howland, "Image registration underwater for fluid flow measurements and mosaicking", in Proc. of the MTS/IEEE OCEANS Conf., vol. 3, pp. 15291534, 2000

[9] S.M. Pizer, E.P. Amburn, J.D. Austin, R. Cromartie, A. Geselowitz, B.H. Romeny, J.B. Zimmerman and K. Zuiderveld, "Adaptive histogram equalization and its variations," Computer Vision, Graphics and Image Processing, no. 39, pp. 355-368, 1987.

[10] R. Eustice, O. Pizarro, H. Singh, J. Howland "UWIT- underwater image toolbox for optical image processing and mosaicking in MATLAB," in Proceedings of the International Symposium on Underwater Technology, pp. 141-145, 2002.

[11] K. Zuiderveld, "Contrast Limited Adaptive Histogram Equalization," Graphics Gems IV, P. Heckbert (ed.), Academic Press, 1994.

[12] A.V. Oppenheim, R.W. Schafer and T.G. Stockham Jr., "Non-linear filtering of multiplied and convolved signals", Proc. IEEE, vol. 56, no. 8, pp. 1264-1291, 1968.

[13] M. Petrou and P. Bosdogianni, "Image Processing: the Fundamentals", John Wiley \& Sons, 1999.

[14] Y. Rzhanov, L. Linnett, and R. Forbes "Underwater Video Mosaicing for Seabed Mapping", in Proc. of the IEEE Conf. on Image Processing, vol. 1, pp. 224-227, 2000. 